\title{
The Telescope Array experiment: Status and Prospects
}

H.Tokuno $^{\text {a }}$, T.Abu-Zayyad ${ }^{\text {b }}$, R.Aida ${ }^{\mathrm{c}}$, M.Allen ${ }^{\mathrm{b}}$, R.Azuma ${ }^{\mathrm{a}}$, E.Barcikowski ${ }^{\text {, J.W.Belz }}{ }^{\text {, T.Benno }}{ }^{\text {, D.R.Bergman }}$, S.A.Blake ${ }^{\text {, }}$, O.Brusova $^{\text {b }}$, R.Cady ${ }^{\text {, }}$, B.G.Cheon ${ }^{\mathrm{f}}$, J.Chiba ${ }^{\mathrm{g}}$, M.Chikawa ${ }^{\mathrm{d}}$, E.J.Cho ${ }^{\mathrm{f}}$, L.S.Cho ${ }^{\mathrm{h}}$, W.R.Cho ${ }^{\mathrm{h}}$, F.Cohen ${ }^{\mathrm{i}}$, K.Doura ${ }^{\mathrm{d}}$, C.Ebeling ${ }^{\mathrm{b}}$, H.Fujii ${ }^{\mathrm{j}}$, T.Fujiik, T.Fukuda $^{\mathrm{a}}$, M.Fukushima, ${ }^{1, \mathrm{~m}}$, D.Gorbunov ${ }^{\mathrm{n}}$, W.Hanlon ${ }^{\mathrm{b}}$, K.Hayashi ${ }^{\mathrm{a}}$, Y.Hayashi $^{\mathrm{k}}$, N.Hayashida ${ }^{\mathrm{i}}$, K.Hibino ${ }^{\mathrm{o}}$, K.Hiyama ${ }^{\mathrm{i}}$, K.Honda ${ }^{\mathrm{c}}$, G.Hughes ${ }^{\mathrm{e}}$,

T.Iguchi $^{a}$, D.Ikeda ${ }^{i}$, K.Ikuta ${ }^{c}$, S.J.J.Innemee ${ }^{\mathrm{e}}$, N.Inoue ${ }^{\mathrm{p}}$, T.Ishii ${ }^{\mathrm{c}}$, R.Ishimori ${ }^{\mathrm{a}}$, D.Ivanov ${ }^{\mathrm{e}}$, S.Iwamoto ${ }^{\mathrm{c}}$, C.C.H.Jui ${ }^{\mathrm{b}}$, K.Kadota ${ }^{\mathrm{q}}$, F.Kakimoto ${ }^{\mathrm{a}}$, O.Kalashev ${ }^{\mathrm{n}}$, T.Kanbe ${ }^{\mathrm{c}}$, H.Kang ${ }^{\mathrm{r}}$, K.Kasahara ${ }^{\mathrm{s}}$, H.Kawai ${ }^{\mathrm{t}}$, S.Kawakami ${ }^{\mathrm{k}}$,

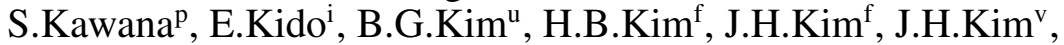
A.Kitsugi ${ }^{i}$, K.Kobayashi ${ }^{g}$, H.Koers ${ }^{\text {w }}$, Y.Kondo ${ }^{\mathrm{i}}$, V.Kuzmin ${ }^{\mathrm{n}}$, Y.J.Kwon ${ }^{\text {h }}$, J.H.Lim ${ }^{\mathrm{r}}$, S.I.Lim ${ }^{\mathrm{u}}$, S.Machida ${ }^{\mathrm{a}}$, K.Martens ${ }^{\mathrm{x}}$, J.Martineau ${ }^{\mathrm{b}}$, T.Matsuda ${ }^{\mathrm{j}}$, T.Matsuyama $^{\mathrm{k}}$, J.N.Matthews ${ }^{\mathrm{b}}$, M.Minamino ${ }^{\mathrm{k}}$, K.Miyata ${ }^{\mathrm{g}}$, H.Miyauchi $^{\mathrm{k}}$, Y.Murano $^{\mathrm{a}}$, T.Nakamura ${ }^{\mathrm{y}}$, S.W.Nam ${ }^{\mathrm{u}}$, T.Nonaka ${ }^{\mathrm{i}}$, S.Ogio $^{\mathrm{k}}$, M.Ohnishi ${ }^{\mathrm{i}}$, H.Ohoka ${ }^{\mathrm{i}}$, T.Okudak ${ }^{\mathrm{k}}$, A.Oshimak ${ }^{\mathrm{k}}$, S.Ozawa ${ }^{\mathrm{s}}$, I.H.Park ${ }^{\mathrm{u}}$, D.Rodriguez ${ }^{\mathrm{b}}$,

S.Y.Roh ${ }^{v}$, G.Rubtsov ${ }^{\mathrm{n}}$, D.Ryu ${ }^{\mathrm{v}}$, H.Sagawa ${ }^{\mathrm{i}}$, N.Sakurai ${ }^{\mathrm{i}}$, L.M.Scott ${ }^{\mathrm{e}}$, P.D.Shah ${ }^{\mathrm{b}}$, T.Shibata ${ }^{\mathrm{i}}$, H.Shimodaira ${ }^{\mathrm{i}}$, B.K.Shin ${ }^{\mathrm{f}}$, J.D.Smith ${ }^{\mathrm{b}}$, P.Sokolsky ${ }^{\mathrm{b}}$, T.J.Sonley ${ }^{\mathrm{b}}$, R.W.Springer ${ }^{\mathrm{b}}$, B.T.Stokes ${ }^{\mathrm{e}}$, S.R.Stratton ${ }^{\mathrm{e}}$, S.Suzuki ${ }^{\mathrm{j}}$, Y.Takahashi ${ }^{i}$, M.Takeda $^{\mathrm{i}}$, A.Taketa $^{\mathrm{i}}$, M.Takita ${ }^{\mathrm{i}}$, Y.Tameda $^{\mathrm{a}}$, H.Tanaka $^{\mathrm{k}}$, K.Tanaka ${ }^{\mathrm{z}}$, M.Tanaka ${ }^{\mathrm{j}}$, J.R.Thomas ${ }^{\mathrm{b}}$, S.B.Thomas ${ }^{\mathrm{b}}$, G.B.Thomson ${ }^{\mathrm{e}}$, P.Tinyakov $^{\mathrm{a}, \mathrm{w}}$, I.Tkachev ${ }^{\mathrm{n}}$, T.Tomida ${ }^{\mathrm{c}}$, R.Torii ${ }^{\mathrm{i}}$, S.Troitsky $^{\mathrm{n}}$, Y.Tsunesada ${ }^{\mathrm{a}}$,

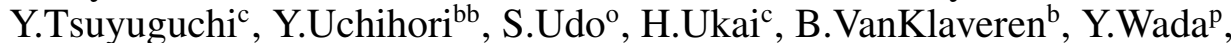
M.Wood ${ }^{\mathrm{b}}$, T.Yamakawa ${ }^{\mathrm{i}}$, Y.Yamakawa ${ }^{\mathrm{i}}$, H.Yamaoka ${ }^{\mathrm{j}}$, J.Yang $^{\mathrm{u}}$, S.Yoshida $^{\mathrm{t}}$, H.Yoshii ${ }^{\mathrm{cc}}$ and Z.Zundel ${ }^{\mathrm{b}}$

${ }^{a}$ Tokyo Institute of Technology, Meguro, Tokyo, Japan

${ }^{b}$ University of Utah, High Energy Astrophysics Institute, Salt Lake City, Utah, USA

${ }^{c}$ University of Yamanashi, Interdisciplinary Graduate School of Medicine and Engineering, Kofu, Yamanashi, Japan

${ }^{d}$ Kinki University, Higashi Osaka, Osaka, Japan

${ }^{e}$ Rutgers University, Piscataway, USA

${ }^{f}$ Hanyang University, Seongdong-gu, Seoul, Korea

${ }^{g}$ Tokyo University of Science, Noda, Chiba, Japan

${ }^{h}$ Yonsei University, Seodaemun-gu, Seoul, Korea

${ }^{i}$ Institute for Cosmic Ray Research, University of Tokyo, Kashiwa, Chiba, Japan ${ }^{j}$ Institute of Particle and Nuclear Studies, KEK, Tsukuba, Ibaraki, Japan

${ }^{k}$ Osaka City University, Osaka, Osaka, Japan

${ }^{l}$ Institute for Cosmic Ray Research, University of Tokyo, Kashiwa, Chiba, Japan

${ }^{m}$ Institute for the Physics and Mathematics of the Universe, University of Tokyo, Kashiwa, Chiba,

Japan

CP1238, Tours Symposium on Nuclear Physics and Astrophysics - VII

edited by H. Susa, H. Utsunomiya, M. Arnould, S. Galès, T. Motobayashi, and C. Scheidenberger

(C) 2010 American Institute of Physics 978-0-7354-0784-8/10/\$30.00 


\author{
${ }^{n}$ Institute for Nuclear Research of the Russian Academy of Sciences, Moscow, Russia \\ ${ }^{o}$ Kanagawa University, Yokohama, Kanagawa, Japan \\ ${ }^{p}$ Saitama University, Saitama, Saitama, Japan \\ ${ }^{q}$ Tokyo City University, Setagaya-ku, Tokyo, Japan \\ ${ }^{r}$ Pusan National University, GeumJeong-gu, Busan, Korea \\ ${ }^{s}$ Waseda University, Advanced Research Institute for Science and Engineering, Shinjuku-ku, Tokyo, \\ Japan \\ ${ }^{t}$ Chiba University, Chiba, Chiba, Japan \\ ${ }^{u}$ Ewha Womans University, Seodaaemun-gu, Seoul, Korea \\ ${ }^{v}$ Chungnam National University, Yuseong-gu, Daejeon, Korea \\ ${ }^{w}$ University Libre de Bruxelles, Brussels, Belgium \\ ${ }^{x}$ University of Tokyo, Institute for the Physics and Mathematics of the Universe, Kashiwa, Chiba, \\ Japan \\ ${ }^{y}$ Kochi University, Kochi, Kochi, Japan \\ ${ }^{z}$ Hiroshima City University, Hiroshima, Hiroshima, Japan \\ ${ }^{a}$ Institute for Nuclear Research of the Russian Academy of Sciences, Moscow, Russia \\ ${ }^{b b}$ National Institute of Radiological Science, Chiba, Chiba, Japan \\ ${ }^{c c}$ Ehime University, Matsuyama, Ehime, Japan
}

\begin{abstract}
The Telescope Array is a detector of extensive air shower produced by ultra High energy cosmic ray. This detector is located on Utah, USA. The construction have been completed and the full operation has been running from March 2008. In this talk, the status of observation and our prospects are described.
\end{abstract}

Keywords: Extensive Air Shower, Ultra High Energy Cosmic Rays, PACS: 96.50.sd,

\title{
INTRODUCTION
}

The Telescope Array (TA) is a hybrid detector consists of a surface detector (SD) array and fluorescence detectors (FDs) [1,2]. This hybrid detector is observing extensive air showers (EASs) to measure the energy spectrum, anisotropy and composition of Ultra High Energy Cosmic Rays (UHECRs). From these information, we investigate origins of UHECRs. This detector is located in Utah, USA (39.3 N, $112.9 \mathrm{~W})$ with an average altitude of $1400 \mathrm{~m}$. The SD array consists of 507 detectors, each $3 \mathrm{~m}^{2}$ in area, with $1.2 \mathrm{~km}$ spacing, and its total area is $700 \mathrm{~km}^{2}$. Three FD stations are surrounding the SD array, and observe the night sky above the SD array. The field of view of these FD stations is 108 degree (114 degree for the third station) in azimuth and 3-31 degree in elevation. More details of our detector configuration are described in [1,2]. The detector construction has been completed in March 2008, and the hybrid observation with the full configuration has been running since that time.

UHECRs will interact with the cosmic microwave background, and a cutoff in their energy spectrum is expected around the energy $10^{19.5} \mathrm{eV}$ by Greisen, Zatsepin and Kuzmin [3, 4]. This expected cutoff structure is called GZK cutoff. HiRes and Auger reported that there is a cut-off in their observed energy spectrum $[5,6]$. In contrast, the AGASA result shows that there are 11 events above $10^{20} \mathrm{eV}$ [7]. These three experimental groups claim their systematic uncertainty in energy determination is about $20 \%$ respectively [5-7]. The rescaling energy spectra of AGASA, HiRes, and Auger within their uncertainties are in agree well below $10^{20} \mathrm{eV}$ [8]. It seems that the inconsistency is due to the systematic uncertainties of these experiments in energy determination. To 
resolve this situation, we compare the response of AGASA type detector (plastic scintillation counter array) with that of HiRes type detector (air fluorescence light detector) directly using observed same EAS events.

\section{OBSERVATION STATUS}

Number of installed SD was 503 in March 2008. Four additional detectors were installed in November 2008, and totally 507 SDs are running from that time. Our SD array is taking data with three sub-arrays from March 2008, and all sub-arrays were integrated as a whole array in November 2008. This integration reduced array boundary, and increases number of analyzable events. The trigger condition of the array is three adjacent detectors with more than three particle's equivalent signal. The average trigger rate is 20 triggers/hour with this trigger condition. The average number of available SD is more than $98 \%$, and down time of SD observation is less than $4 \%$. We are online monitoring SD performances: detector gain, accuracy of internal clock, charge level of battery, trigger rate, temperature and humidity inside the detectors, and accuracy of GPS clock. This online monitoring system help us to keep a stable DAQ run.

The first FD station and a half of the second station are running from May 2008, and the another half of the second station has been started from December 2008. The trigger rate for a station is about $2 \mathrm{~Hz}$ with $6 \%$ dead time. The total running time of the first and second station is 2370, 2025 hours respectively at November 2009. From May 2009, the second FD station is operated remotely from the first station. We monitor FD performances: PMT gain, mirror reflectance, window transmittance [9]. For atmospheric monitoring, we employ a Central Laser Facility located on the same distance from the three FD stations, LIDAR (LIght Detection And Ranging), and Infrared camera for cloud monitoring $[10,11]$. The FDs at the third station were moved from HiRes-I, and these detectors have been running from December 2008. The FDs at the third station provide preliminary results, which are consistent with HiRes-I experimental results (C.C.H.Jui et al. oral presentation on ICRC2009).

We reported our recent observation status also in International Cosmic Ray Conference 2009 (The Proceedings will be available online soon).

\section{PROSPECTS}

Our SD array and Mono-FDs will obtain AGASA equivalent exposure by 2010 summer. Expected number of events is about 800 with the primary energy above $10^{19} \mathrm{eV}$ with an assumption of AGASA flux. One hundred events of them will be detected by SD array and FDs coincidentally. Using these events, we are studying the differences of estimated energy between SD and FDs. To study the differences in detail, we employ new equipments for SD DAQ system and FD calibration and atmospheric monitoring. For example, SD records waveforms of PMT output with $50 \mathrm{MHz}$ sampling FADC. Using recorded waveforms, we can study the detail of shower front structure and delayed particles, which may cause uncertainties of energy determination of AGASA [12]. Meanwhile, various equipments will be applied to calibrate FDs optics and monitor 
for transparency of the atmosphere $[10,11,13,14]$. They are monitoring atmospheric conditions continuously during observation periods. Estimation accuracy of FD aperture will also be studied carefully using these monitor data. In addition, $40 \mathrm{MeV}$ electron beam system on the front of the first station is being prepared for End to End calibration which includes air fluorescence yield to electronics performances [15]. This End to End calibration will reduce systematic errors of energy determination of FD.

\section{ACKNOWLEDGMENTS}

The Telescope Array experiment is supported by the Ministry of Education, Culture, Sports, Science and Technology-Japan through Kakenhi grants on priority area (431) "Highest Energy Cosmic Rays", basic research awards 18204020(A), 18403004(B) and 20340057(B); by the U.S. National Science Foundation awards PHY-0307098, PHY0601915, PHY-0703893, PHY-0758342, and PHY-0848320 (Utah) and PHY-0649681 (Rutgers); by the Korea Research Foundation (KRF-2007-341-C00020); by the Korean Science and Engineering Foundation (KOSEF, R01-2007-000-21088-0); by the Russian Academy of Sciences, RFBR grants 07-02-00820a and 09-07-00388a (INR), the FNRS contract 1.5.335.08, IISN and Belgian Science Policy under IUAP VI/11 (ULB). The foundations of Dr. Ezekiel R. and Edna Wattis Dumke, Willard L. Eccles and the George S. and Dolores Dore Eccles all helped with generous donations. The State of Utah supported the project through its Economic Development Board, and the University of Utah through the Office of the Vice President for Research. The experimental site became available through the cooperation of the Utah School and Institutional Trust Lands Administration (SITLA), U.S. Bureau of Land Management and the U.S. Air Force. We also wish to thank the people and the officials of Millard County, Utah, for their steadfast and warm supports. We gratefully acknowledge the contributions from the technical staffs of our home institutions and the University of Utah Center for High Performance Computing (CHPC).

\section{REFERENCES}

1. H. Kawai, and et al., Nucl.Phys.B Proc.Suppl 175-176, 221-226 (2008).

2. H. Kawai, and et al., J.Phys.Soc.Jpn. 78, 108-113 (2009).

3. K.Greisen, Phys. Rev. Lett. 16, 748 (1966).

4. T.Zatsepin, and V.A.Kuzmin, JETP Lett. 4, 78 (1966).

5. J. Abraham, and et al., Phys. Rev. Lett. 101, 061101 (2008).

6. R. Abbasi, and et al., Phys. Rev. Lett. 100, 101101 (2008).

7. M. Takeda, and et al., Astropart. Phys. 19, 447-462 (2003).

8. V. Berezinsky, Proc. of 30th ICRC 6, 21-33 (2009).

9. H. Tokuno, and et al., NIM A 601, 364-371 (2009).

10. M. Chikawa, and et al., Proc. of 30th ICRC 5, 1025-1028 (2008).

11. S. Udo, and et al., Proc. of 30th ICRC 5, 1021-1024 (2008).

12. M. Nagano, New Journal of Physics 11, 065012 (2009).

13. J. N. Matthews, and et al., Proc. of 30th ICRC 5, 1157-1158 (2008).

14. H. Tokuno, and et al., Proc. of 30th ICRC 5, 1013-1016 (2008).

15. T. Shibata, and et al., NIM A 597, 61-66 (2008). 